\title{
Corrigendum to Resolutions of homology manifolds, and the topological characterization of manifolds
}

Invent. Math. 72, 267-284 (1983)

\section{Frank Quinn}

Virginia Polytechnic Institute and State University, Department of Mathematics,

Blacksburg, VA 24061, USA

There is an error in Sect. 4 of [1]. Rather than showing that the "last obstruction" is trivial, the proof shows that it defines an invariant (the local index) of connected ENR homology manifolds, which satisfies $i(X) \equiv 1(\bmod 8)$, $i(U)=i(X)$ if $X \supseteq U$ is open, $i(X \times Y)=i(X) i(Y)$, and if $\operatorname{dim}(X) \geqq 5$ or $\operatorname{dim}(X)$ $=4$ and $\partial X$ is a manifold, then $X$ has a resolution if and only if $i(X)=1$. Consequently in the statements of the "characterization of manifolds" and 1.11.3 of [1], "homology manifold" should be replaced by "homology manifold of local index 1". Details will appear elsewhere.

1. Quinn, F.: Resolutions of homology manifolds and the topological characterization of mantfolds. Invent. Math. 72, 267-284 (1983) 